ANNALES

UNIVERSITATIS MARIAE CURIE-SKŁODOWSKA

LUBLIN - POLONIA

VOL. LXX, 1

SECTIO AA

2015

\title{
Sugar-based surfactants as alternative to synthetic ones
}

\author{
Diana Mańko* and Anna Zdziennicka \\ Faculty of Chemistry, Department of Interfacial Phenomena, \\ Maria Curie-Skłodowska University \\ M. Curie-Skłodowskiej Sq. 3, 20-031 Lublin, Poland \\ *e-mail: manko_diana@interia.pl
}

From year to year natural surfactants are gaining ever growing awareness because of their valuable properties (in the absence of negative impact on the environment, as with the use of synthetic surface active agents). In the group of surfactants of natural origin there are surface active agents produced by microorganisms (biosurfactants) or compounds having a natural part (hydrophilic head and/or hydrophobic tail) [1]. This review includes the short characteristic of surfactants with sugar-head group in their structure called sugar-based surfactants. The structure, properties and some applications of these surfactants are described in this paper.

\section{INTRODUCTION}

Nowadays, in connection with the increasing pollution of the environment, numerous studies are carried out in order to find a new group of compounds which could replace surface active agents produced by chemical synthesis due to their high toxicity to ecosystem and difficulty in being degraded in the environment. A potential alternative to these compounds are sugar-based surfactants - amphiphilic surface active agents produced from renewable materials [2]. Their natural origin causes that they are environmentally friendly, biodegradable and non-toxic [3]. The biological and environmental compatibilities are not the only advantages of natural surfactants over synthetic ones because numerous 
papers indicate that in many cases they present better physicochemical properties than compounds produced on the basis of non-renewable resources. Therefore these surfactants are increasingly used in many areas of human life such as industry, agriculture, medicine, everyday life and even in the environment protection. The number and the type of raw materials used to produce sugar-based surfactants are increasing from year to year giving the new possibilities in modeling their properties and expanding the scope of their applications.

\section{GENERAL CONSIDERATIONS}

Surface active agents are amphiphilic compounds consisting of two different parts: a polar (hydrophilic) head and a non-polar (hydrophobic) tail. Therefore they can accumulate at different interfaces and decrease interfacial tension at the boundary between them. Moreover, surfactants at concentration above their Critical Micelle Concentration (CMC) form aggregate structures co-called micelles. These two tendencies to adsorb at different interfaces and to micellization process give them a valuable technological properties (such as dispersing, foaming, emulsifying, wetting properties) and the opportunity to be used in both everyday life and many industries [4].

There are two main groups of surface active agents: synthetic surfactants and natural ones. Synthetic surfactants are produced by chemical reactions from non-renewable resources, mainly from petroleum, natural gas or coal, whereas in the preparation of natural surfactants no organic synthesis should be involved at any stage of the process and they are synthesized from natural substrates, such as plant oils, animal fats, sugars, proteins [4]. Among natural surfactants these based on sugars are very promising from ecological and technological point of view.

Sugar-based surfactants are surface active agents with sugar group in their structure. The kind of sugar group in the molecule of surfactant depends on the type of raw material used for its production. The number of natural products is large but only some of them can be are proper for being transformed into surfactants [5]. These starting materials are generally plentiful, inexpensive, reproducible and biocompatible [6] and their type determines not only the construction of final sugar surface active agent but also it impacts on physicochemical and ecological properties of the obtained compound. 
Sugar-based surfactants can be derived from the sugar raw materials occurring in the form of monomeric (such as glucose, fructose, sorbitol), dimeric (such as sucrose, lactose) or polymeric (such as starch, cellulose, pectin, chitin, polysaccharides, dextrins) compounds as well as from sugars which remain as post-production wastes $[5,7]$. At the same time, these materials may constitute the hydrophilic head in the molecule of obtained surface active agent which is linked glycosidically with the alkyl moiety of the fatty alcohol or which is linked with the rest of the fatty acid by the ester, amine or amide bond [8].

The type of chemical bond existing between the hydrophilic and hydrophobic parts of the surfactant molecule has an effect on its properties. The presence of amide group in the molecule reduces the solubility of the surfactant in water and gives a stable connection between the head and tail of the surfactant characterizing high resistance to acidic and basic hydrolysis. Moreover, the amide bond is easily degraded because living organisms have enzymes capable of breaking it down. The ether groups form a strong bond not easily degraded in nature in contrast to the ester groups which form a weaker bond, useful and flexible which can be easily hydrolyzed [5]. The hydrophilic group could consist of many the same or different sugar units and the hydrophobic part could consist of one, two and even more alkyl chains with the same or different lengths [8]. The representative structure of one of the most popular sugar surfactants used in practice practice - 2-(decyloxy)-6-(hydroxylmethyl) oxane-3,4,5-triol (n-oktyl- $\beta$-D-glucopiranoside, OGP), which is composed of one molecule of glucose as a head linked glycosidically with the straight, alkyl chain constituting the non-polar tail [9], is shown below in Figure 1.

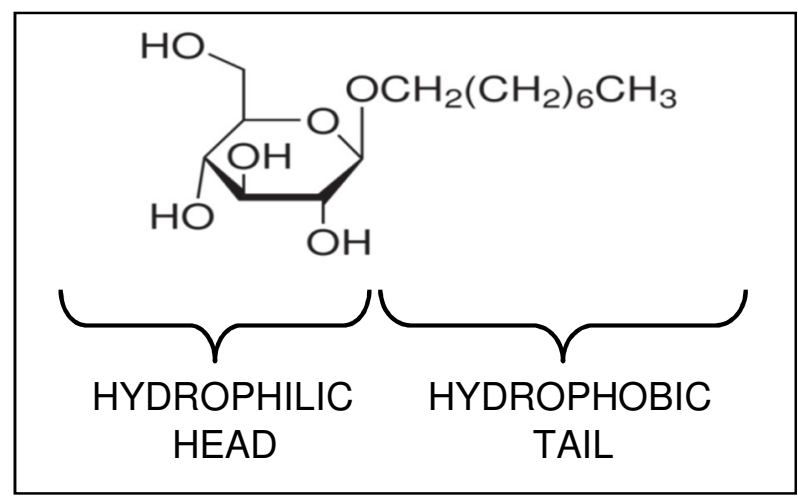

Fig. 1. The representative structure of sugar-based surfactant - OGP. 
A wide group of sugar-based surfactants can be includes among others, alkyl glycosides, alkyl thio-glycosides, alkyl maltosides and their homologues or derivatives, betaines (zwitter-ionic surfactants), gemini (compounds with two monomeric surfactant molecules linked by a spacer chain) and bolas (compounds with two polar groups linked to both ends of a long alkyl tail) surfactants. Among them those based on glucosides, especially alkyl polyglucosides (APGs), are often used [3, 10].

As mentioned at the beginning, surfactants derived from natural products are non-toxic and have a high rate of biodegradation because they can be composed of a variety of ingredients commonly occurring in the environment. These compounds have high surface activity because even at low concentration they are very effective in diminishing the surface tension of water and the water/oil interface tension [3]. A large number of sugar-based surfactants are also characterized by high values of CMC, for example this value for n-oktyl- $\beta$-D-glucopiranoside at $25^{\circ} \mathrm{C}$ determined by Shinoda et al. is equal to $2.4 \cdot 10^{-2} \mathrm{M}$.

In comparison to chemical surfactants these with sugars as a polar head are more stable and sustainable. Moreover they are no-irritating and mild for skin, mucous membranes and eyes and therefore they are very attractive for personal care products, especially for facial skin care and those used for the delicate skin of children $[10,12]$. Besides, depending on the structure surfactants have characteristic features. For instance, alkyl polyglucosides are stable at high $\mathrm{pH}$ and sensitive to low $\mathrm{pH}$ in which they hydrolyze, They do not exhibit clear relationship between solubility and temperature as for most typical nonionic compounds including poly(ethylene oxide) surfactants [1].

Surface active agents derived from natural products find numerous application not only as detergents or antifoaming agents but they also play an important role in the field of technology, biotechnology, medicine and biology. Sugar-based surfactants can be used in the separation process called micellar enhanced ultrafiltration (MEUF) which is used for the separation of low molecular weight pollutants from the aqueous phase using a surfactant at the $\mathrm{CMC}$. This process involves increasing the size of pollutant molecules by closing them inside the surfactant micelles. So the obtained complexes of pollutants and surfactant are retained on the surface of the ultrafilter membrane and thus allow to separate them from the solution [13].

Among other technical applications it is also worth mentioning the possibilities of the use sugar surfactant microemulsions in practice. Microemulsions consist of water, oil, surfactant and/or co-surfactant at 
different mass ratios and these systems can be drug delivery vehicles as well as microreactors for some organic reactions. Different kinds of surfactants are proposed to create and stabilize the microemulsion used in various fields of industry e.g. in cosmetic industry, but surfactants used in food-systems have to satisfy the additional requirements such as defined solubility and non-toxicity. Biodegradable surfactants such as sugar-based surfactants are of special interest in this process [14].

Surfactants based on sugars are commonly used in biotechnology and biology, especially in protein solubilization and membrane research [15]. In membrane studies many of them are used for isolation, characterization and crystallization of a large number of proteins because of their high values of $\mathrm{CMC}$, which simplifies their removal by dialysis. Moreover, they can be also used in the process of reconstitution of membrane proteins with lipids in the biomimetic systems [16, 17].

The research shows that the surfactants belonging to the group of alkyl polyglucosides are very effective in solubilization of hydrocarbons and organochlorine compounds, in the process of oil pollution washing from the soil. They are also popular in use in composition of pesticides and agrochemicals [18].

Many of the surfactants based on simple sugars, as for example decyl glucoside, are components of cleaning products and cosmetic preparations (shampoos, shower gels, bubble baths, shaving foams) because they show high efficiency as washing, cleaning, wetting and foaming compounds. Simultaneously they are low-irritating, very mild and gentle for the skin and they maintain skin balance without dryness. These surfactants are also interesting for laundry and dishwashing detergents [10, 12].

This class of surfactants finds some applications in medicine and pharmacy. They have been shown to become drug vehicles (like e.g. the amphiphilic dextran derivatives), they also can replace other, more toxic and allergenic surfactants in the protein formulations (like e.g. trehalose fatty acid esters), some of them possess antibacterial activities (like sugarbased gemini surfactants) or like chemically pure alkyl polyglucoside they can be used to reconstitute biologically active proteins as they have non-denaturing properties $[19,20]$.

Systematic studies regarding further application of sugar surfactants, especially in medicine and technology, are still of a special interest and intensively carried out. 


\section{CONCLUSIONS}

A new family of surfactants based on simple sugars and obtained from naturally occurring and renewable materials is promising as for the future. Because of their advantageous characteristics: biodegradability, low toxicity, environmental compatibility, cheapness, excellent washing and cleaning abilities as well as high surface activity there is a growing interest in considering them as a potential alternative to compounds obtained by chemical synthesis.

Surfactants derived from naturally occurring products are potential substitutes for synthetic ones in several industrial processes, such as lubrication, wetting, making emulsion, foaming, as well as in food, cosmetic, pharmaceutical industries and also in biology and biotechnology. They have already been successfully used in many technical applications.

\section{ACKNOWLEDGEMENTS}

We would like to thank Prof. Bronisław Jańczuk at the Maria CurieSkłodowska University for his valuable contributions to throughout our research activities.

\section{REFERENCES}

[1] K. Holmberg, Curr. Opin. Colloid Interface Sci., 6, 148-159, (2001).

[2] M. E. Abdel-Raouf, A. M. Abdul-Raheim and A. A. A. Abdel-Azim, J. Surfact. Deterg., 14, 113-121, (2011).

[3] C. C. Ruiz (Ed.), Sugar-Based Surfactants: Fundamentals and Applications, CRC Press, (2008).

[4] M. J. Rosen, Surfactants and interfacial phenomena, John Wiley \& Sons, Inc., Hoboken, New Jersey, (2004).

[5] P. S. Piispanen, Synthesis and characterization of surfactants based on natural products, Kungl Tekniska Högskolan, Stockholm, (2002).

[6] K. Shinoda, A. Carlsson and B. Lindman, Adv. in Coll. and Interface Sci., 64, 253-271, (1996). 
[7] M. Kjellin and I. Johansson, Surfactants from renewable resources, John Wiley \& Sons, (2010).

[8] C. Stubenrauch, Curr. Opin. Colloid Interface Sci., 6, 160-170, (2001).

[9] D. Mańko, A. Zdziennicka and B. Jańczuk, Colloids Surf., B, 114, 170-176, (2014).

[10] M. T. Garcia, I. Ribosa, E. Campos and J. Sanchez Leal, Chemosphere, 35, 545-556, (1997).

[11] K. Shinoda, T. Yamaguchi and R. Hori, Bull. Chem. Soc. Jpn., 34, 237-241, (1961).

[12] T. Forster, H. Hensen, R. Hofmann and B. Salka, Cosmet. Toilet., 110, 23-29, (1995).

[13] R. Bade and S. H. Lee, J. Water Sustainability, 1, 85-102, (2011).

[14] N. Garti, Curr. Opin. Colloid Interface Sci., 8, 197-211, (2003).

[15] R. M. Muruganathan, R. Krustev, H.-J. Müller, H. Möhwald, B. Kolaric and R. v. Klitzing, Langmuir, 20, 6352-6358, (2004).

[16] S. Morandat and K. El Kirat, Colloids Surf., B, 55, 179-184, (2007).

[17] R. Zhang, P. A. Marone, P. Thiyagarajan and D. M. Tiede, Langmuir, 15, 7510-7519, (1999).

[18] S. Pastewski, A. Bardachowska and K. Mędrzycka, Inż. Ap. Chem., 5, 84-85, (2011).

[19] B. S. Sekhon, J. Pharm. Technol. Res. Manag., 1, 11-36, (2013).

[20] Ribosa, J. Sanchez Leal , F. Comelles and M. T. Garcia, J. Colloid Interface Sci., 187, 443-446, (1997).

\section{CURRICULA VITAE}

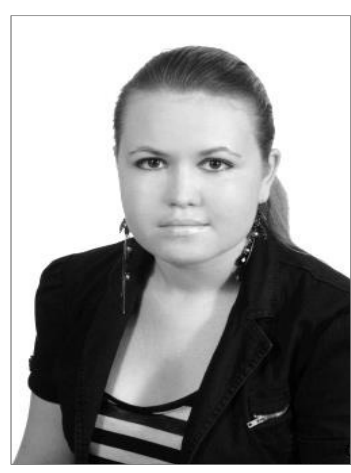

Diana Mańko. Born in Radzyń Podlaski (Poland) in 1988. Graduated from Maria Curie-Skłodowska University in Lublin (2012, Department of Theoretical Chemistry). Since 2012 she has been a Ph.D. student in the Department of Interfacial Phenomena at UMCS. Currently she is carrying out research on physicochemical properties of water solutions of sugar-based surfactants, biosurfactants and their mixtures with different additives. 


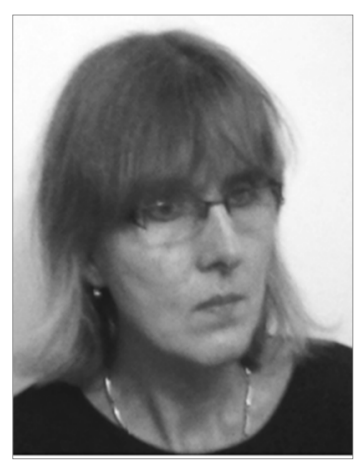

Anna Zdziennicka. Graduated from Maria CurieSktodowska University in Lublin (1988, Faculty of Mathematics, Physics and Chemistry). Received her Ph.D. degree in 1996 in physical chemistry from UMCS. She works in the Department of Interfacial Phenomena in the Faculty of UMCS. Her research work is largely concentrated on physicochemistry of the surface and interface phenomena. She is a coauthor of 90 publications. 